\title{
Research of PROFIBUS PA's integration in PROFINET IO
}

\author{
Zhijia Yang ${ }^{1, a^{*}}$, Zhongsheng $\mathrm{Li}^{1,2, \mathrm{~b}}$, Feng Qiao ${ }^{2}$ and Minghui Min ${ }^{1}$ \\ ${ }^{1}$ Shenyang Institute of Automation, Chinese Academy of Sciences, Shenyang 110016, China \\ ${ }^{2}$ Shenyang Jianzhu University, 9 Hunnan St., Shenyang 110168, China \\ ayang@sia.ac.cn, blizhongsheng@sia.ac.cn
}

Keywords: Industrial Ethernet, PROFINET, PROFIBUS PA, Gateway, Process Control

Abstract. In order to study the integration technology of field bus and industrial Ethernet, a scheme of gateway between PROFINET IO and PROFIBUS PA is proposed. Employing Siemens EB200 IO Evaluation Board to access PROFINET IO network, the gateway is implemented with a PROFIBUS $\mathrm{PA}$ as an external connection driver. Correlated software packages are designed to realize the mutual mapping of data models and network functions between the two protocols. The function test and performance test prove the feasibility of the gateway. It also shows good compatibility with GSD engineering systems.

\section{Introduction}

PROFIBUS PA fieldbus technology which in accordance with IEC61158 standard is designed for process control applications. These years with the development of real-time industrial Ethernet PROFINET IO technology and the advantages it showed make it particularly important to study the gateway technology of PROFINET IO and PROFIBUS PA.

By analyzing the PROFIBUS protocol, literature [1] in the perspective of protocol architecture demonstrates the integration possibilities of PROFIBUS system and PROFINET system. Literature [6] by the way of designing a virtual PROFIBUS Master to map a number of PROFIBUS DP slave to Industrial Ethernet, achieved a real sense of PROFIBUS to Ethernet gateway. After that some people have done the similar research to integrate PROFIBUS DP to Industrial Ethernet, including the interface design even achieved the overall design. But there are some big differences in the physical layer and the transmission rate between PROFIBUS PA and PROFINET IO. So the direct integration has some difficulties, previous engineering integration systems are firstly through PROFIBUS DP / PA segment coupler map PROFIBUS PA slaves to PROFIBUS DP system segment for managing. Then access PROFINET IO through the connection equipment. So little research and design implementation directly integrated PROFIBUS PA into Industrial Ethernet. This paper summarizes the research of others in related areas. Designed and implemented a PROFINET IO-PROFIBUS PA gateway and integrated PROFIBUS PA to PROFINET IO directly.

\section{Gateway model analysis and design}

For this kind of research to integrate a kind of field bus into Industrial Ethernet, due to the rate gap between the two networks, current studies usually use cache-based Proxy scheme [1], that make the gateway as a proxy server supplied to industrial real-time Ethernet and fieldbus different access interfaces. In this case it could serve as a manage station of PROFIBUS PA slaves, simultaneously it could access PROFINET IO like an IO device. In this way the integrated system chart is shown in right chart in Fig.1, the left chart is the current used engineering solution. As we can see the current 3-grades integration is a complex system, it contains three different communication networks. In some case our system don't need a PROFIBUS DP subsystem, the new integration solution is simple and effective in process control systems. 


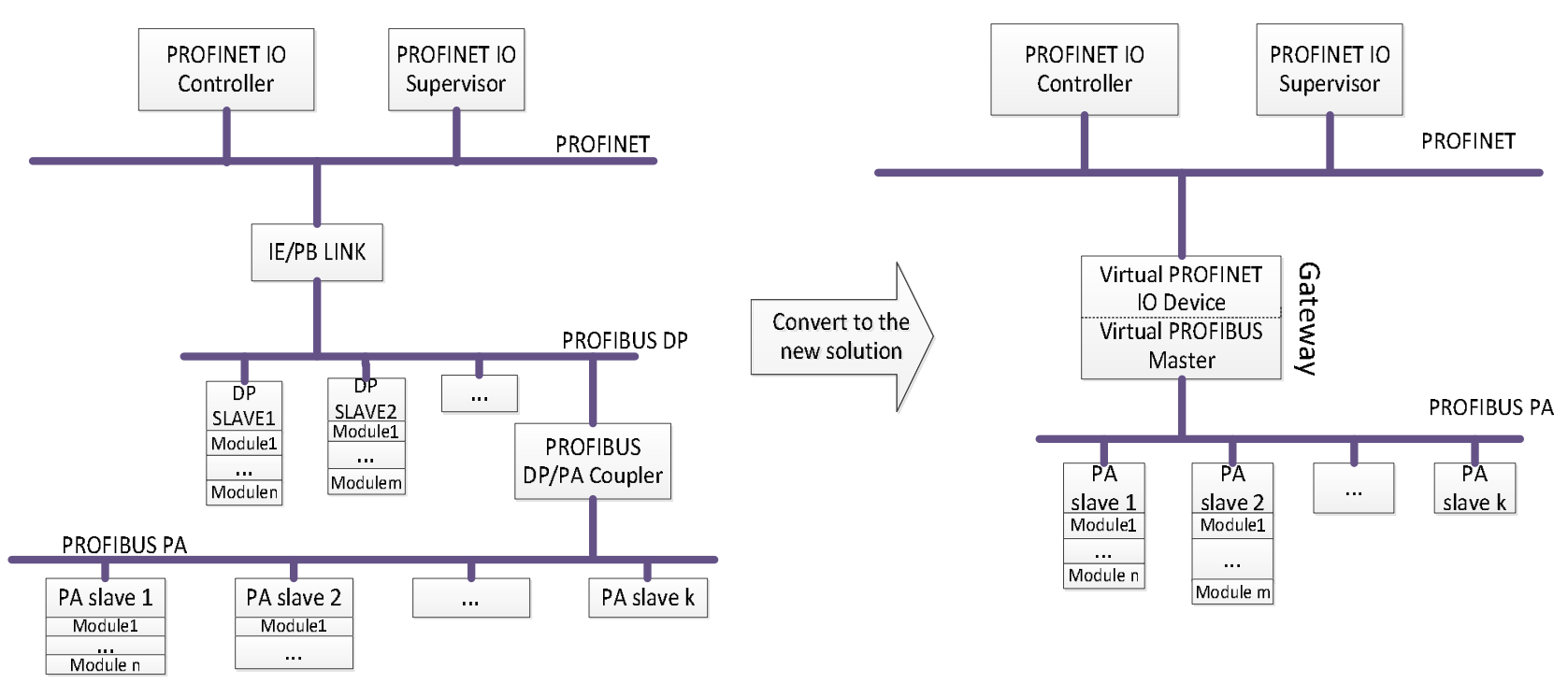

Fig.1 The system contrastive diagram of current engineering and this paper's solution

Protocol Mapping Model. Gateway Design must ensure reliable transmission of real-time IO data and conversions of service requests. It should analyze the two network protocols structure layer by layer and ignore the differences of the two networks. The structure of the protocols conversion is shown in Fig.2.

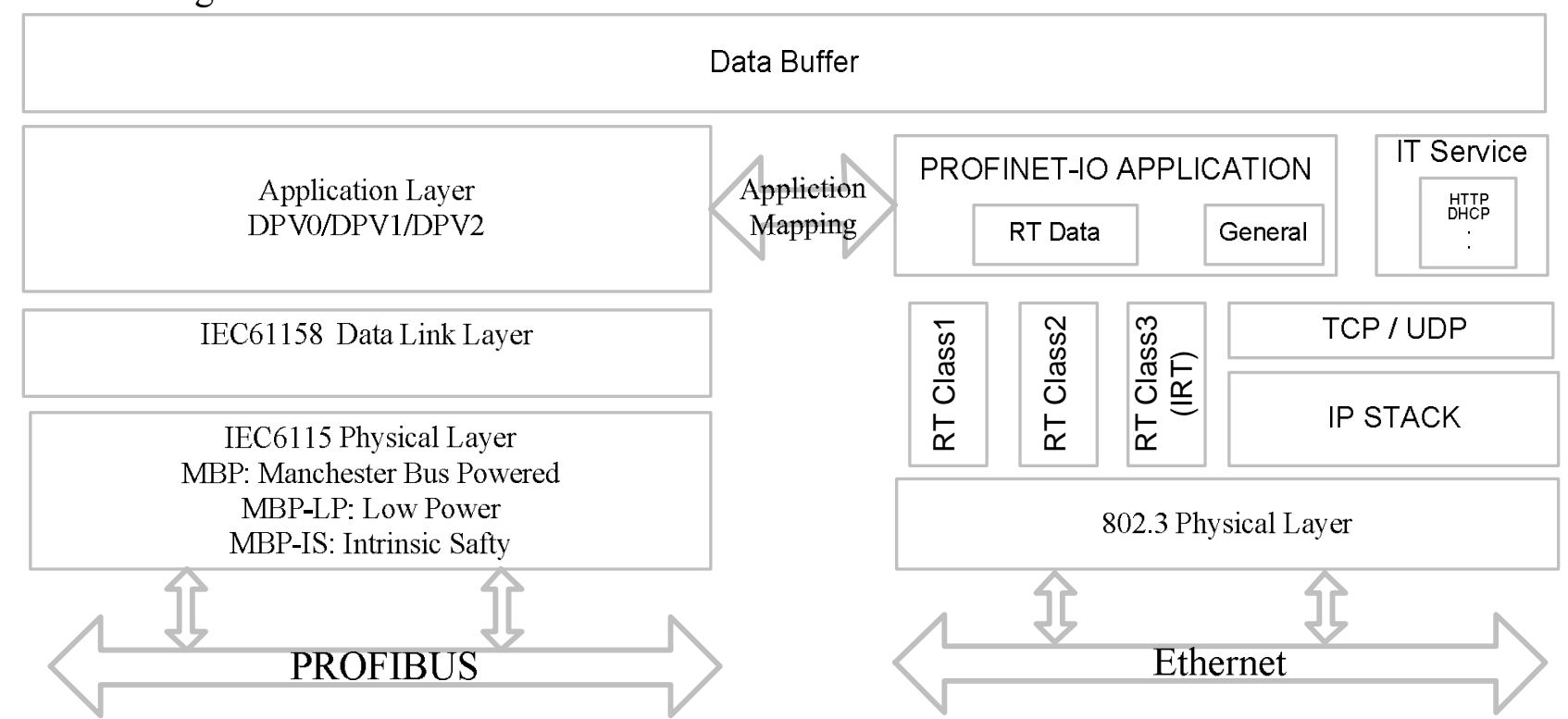

Fig.2 Protocol conversion mapping

Gateway's IO data are stored in the data buffer by the format in PROFINET IO profile, the gateway serve as a PROFIBUS PA master polls slaves once a cycle and take output data from data buffer, encapsulation packet according PROFIBUS PA profile, and sent it to the connected devices on the bus. The input data from PROFIBUS PA slaves should converted to the format meet the PROFINET IO profile and should be put into the data buffer. The high-speed industrial Ethernet read and write IO data from data buffer directly.

Data Mapping Model. Data buffer's data structure model of this gateway device use data model of PROFINET IO's Slot/Subslot structure. There is no hierarchy in Slot/Subslot structures, all accessible individual PROFIBUS PA devices shall be on the same level. The internal modules of a PROFIBUS PA slave should be mapped to Subslots of corresponding Slot. Slot0 is used for controller to read and write the operating parameters of the gateway, it's a device-level access point module, other modules should assign slot from the Slot 1 .

Gateway data model block diagram shown in Fig.3. It contains a PROFINET IO interface module named PNIO inserted into the slot 0 , also includes a module used to access PROFIBUS PA inserted into slot 1. PROFIBUS PA slaves must be mapped to the subsequent slot. As shown in Fig.3 there are 
three PA slaves, each PA slave must be mapped to a slot, respectively slot 2, 3, 4, PROFIBUS PA slave IO data module (PROFIBUS PA device internal module) must be mapped to the corresponding Subslot.

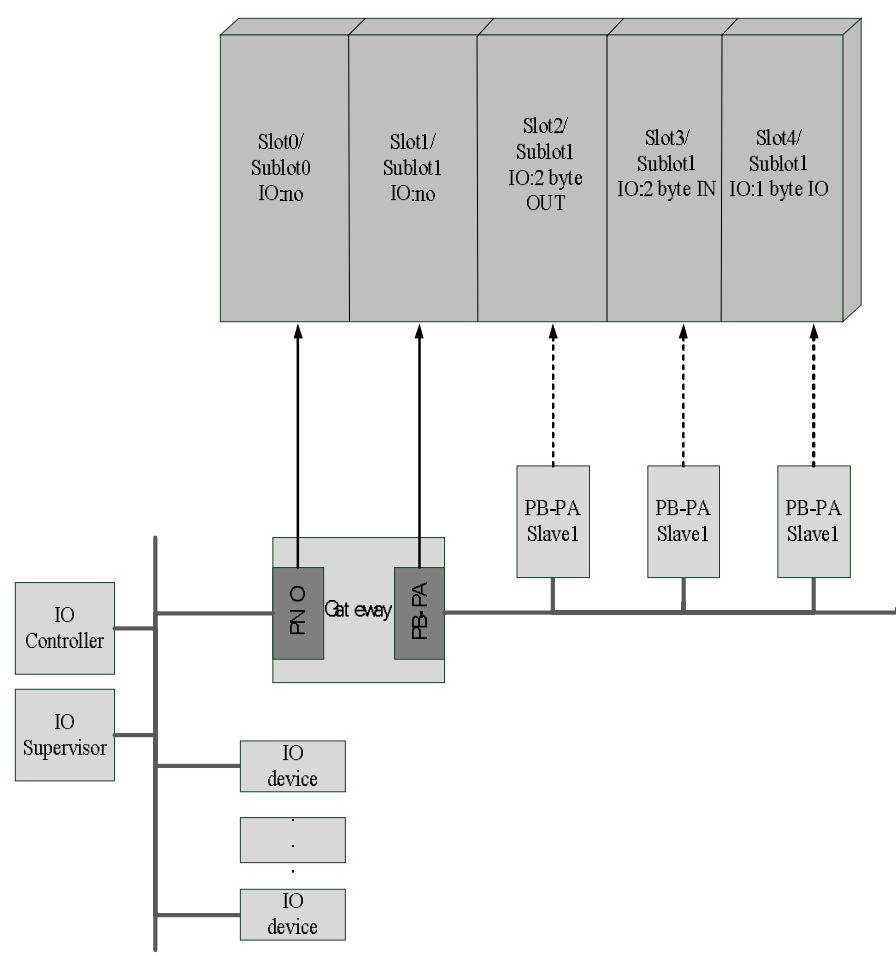

Fig.3 Data mapping model

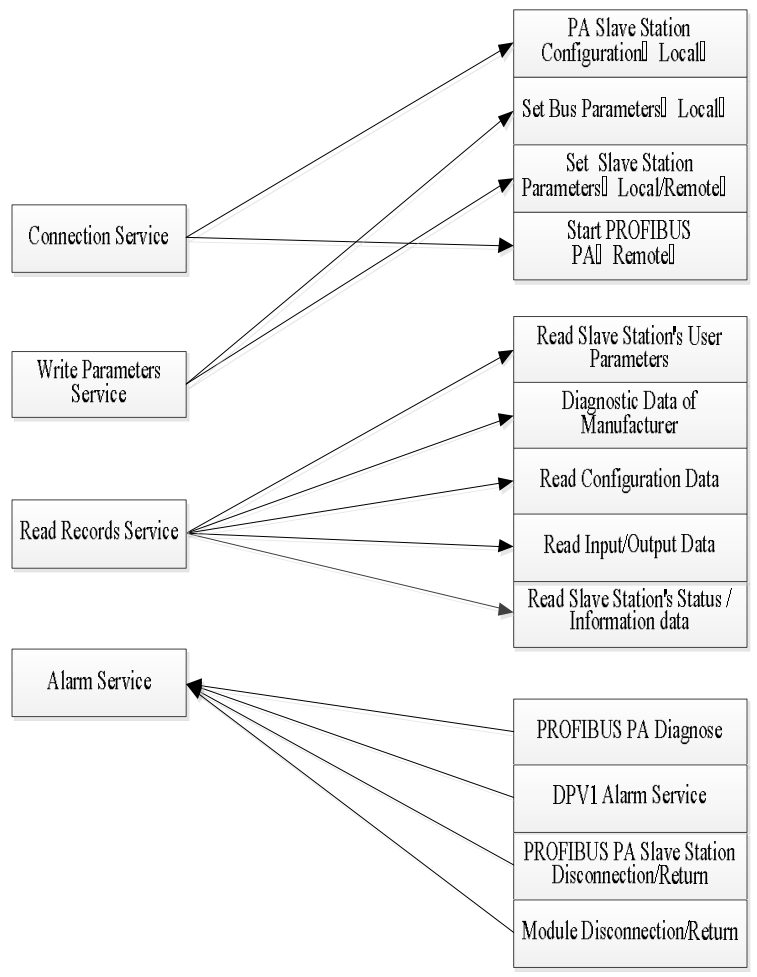

Fig.4 Service mapping model

Service Mapping Model. PROFINET IO service request transmit through the standard TCP / IP protocol, the peer services for both networks must to be mapped to each other, including connection request, reading and writing parameters and alarm services. These services must convert to the corresponding remote services or gateway local services. Service mapping relationship shown in Fig.4, some services are non-essential in some special PROFIBUS PA slave due to the function differences, some doesn't need a peer service.

\section{Gateway implementations}

Gateway implementations include overall structure of the hardware, interface circuit design and protocol mapping software.

Hardware Structure. In literature [3] Lv Y designed a kind of hardware structure used to integrate PROFIBUS PA to PROFINET IO. We reference his solution designed the hardware. For Ethernet interfaces we used Siemens EB200 evaluation board, with two RJ45 connectors, it could access network by various topologies of PROFINET. This board as the main part of the gateway, and then we use FBC0409 communications controller, isolation circuit and SIM-2 PROFIBUS PA bus MAU construct the overall gateway hardware.

Software Design. We have a PROFINET IO device protocol source code package used in EB200 evaluation board, as a result the design work can be simplify. So the three main points are PROFIBUS PA interface driver program design, PROFIBUS soft master protocol design and mapping module design. The given PROFIBET IO source is based eCos embedded real-time operating system, it can be used as the system environment of the Gateway.

PROFIBUS PA interface driver and software implementation need to design software according to PROFIBUS specification, implement cyclic and acyclic with the PA slaves include the functions of slave configuration, parameters setting, alarm and diagnostic mechanism [2]. Mapping module should implement system startup, configuration and protocol data conversion and mapping work during the gateway in normal operation state. The flow chart is shown in Fig.5. 


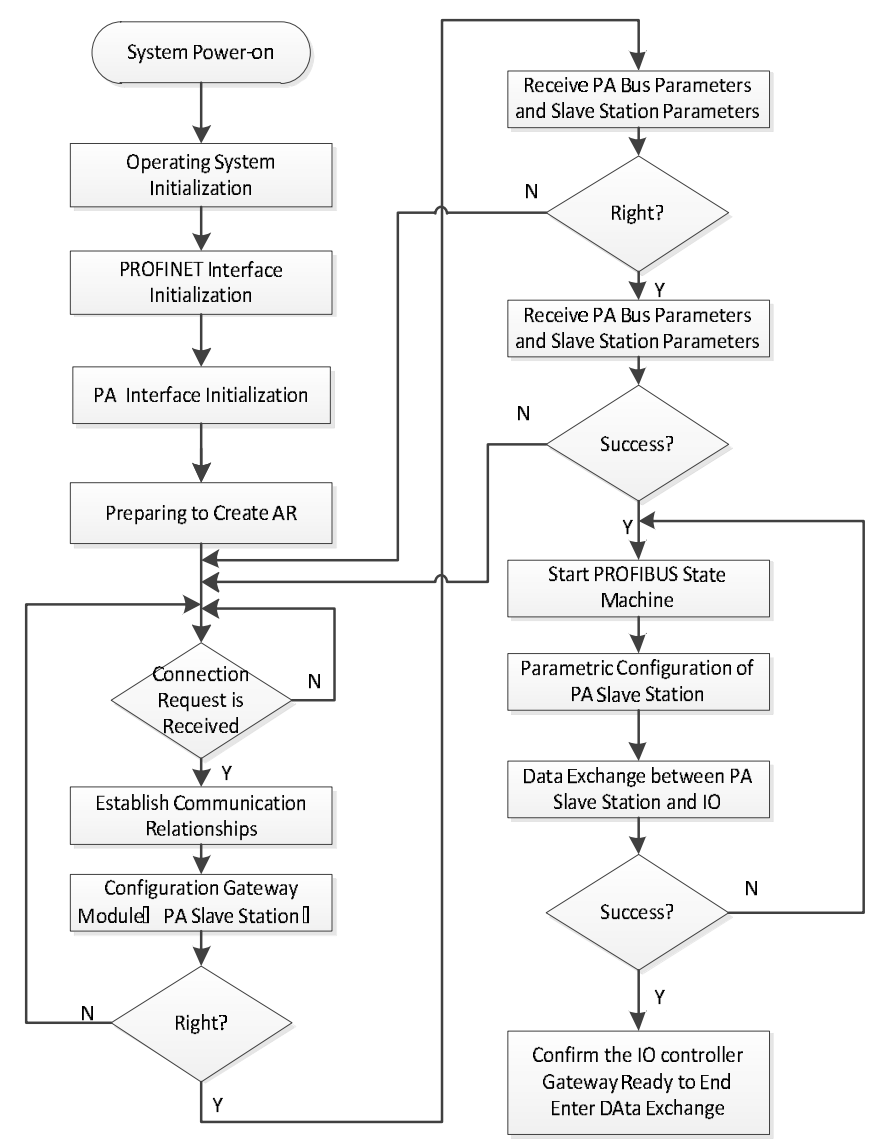

Fig.5 flow chart of gateway software

\section{Engineering integration}

Several device integration techniques apply for fieldbus integration, such as, FDT/DTM, EDD, FDI. In the scope of PROFINET the defined integration standard is GSD in XML language (GSDML). Only this standard is considered here.

In engineering system if a device can be configured we should import its device description file write by GSDML into the engineering tool. In this case the gateway is descripted as an IO device, all PROFIBUS PA slave shall be descripted as an internal module of gateway. The gateway's GSDML file is different in different applications because the different PROFIBUS PA slaves are used. So every time we use the gateway construct a system, we should write a GSDML file for gateway. But it is not convenient enough to users who may not know well about the GSDML file's syntax and how to generate a GSDML file. To simplify engineering process, give users a good engineering interface, we design a GSD tool to help user complete the GSD generation works.

GSD tool are designed according the GSD specification and GSD in XML language (GSDML) standard, it need to convert the PROFIBUS PA device descripted by GSD to gateway's internal modules descripted by GSDML[4,5], the GSD tool GUI are shown in Fig.6.

Use the gateway and this GSD tool the engineering process is shown in the following four steps:

(1) User get the gateway with the corresponding GSD tool;

(2) User get the PROFIBUS PA elements(slave or function modules) which may be used in the application and the corresponding device description file write in GSD style from the vendors of fieldbus devices;

(3) Import all the used PA elements' GSD file into the GSD tool, it would generate a gateway's description file in GSDML style for which can be eat by engineering tool;

(4) Import the GSDML file which generated in step3 to user's engineering tool, that the user's engineering tool would show me a gateway with some PROFIBUS PA modules. After that user could configure the engineering as usual. 


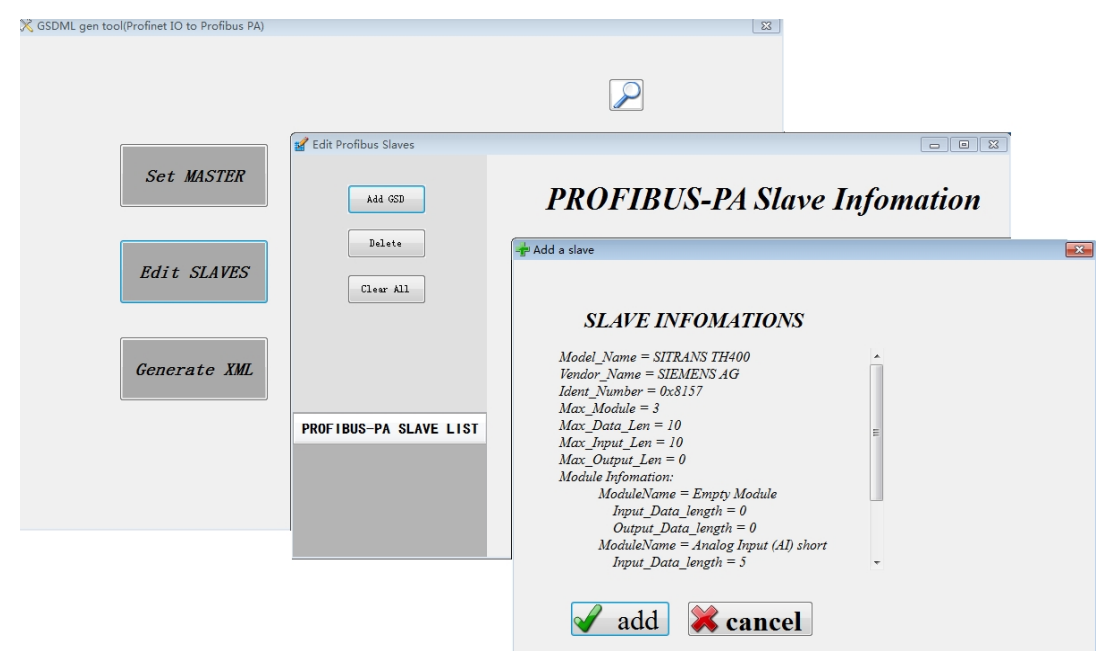

Fig.6 GSD tool GUI

\section{System Testing}

In our laboratory we configure a test system to test our design. The elements of the test system are list in Table 1.

Table 1 Test system configuration

\begin{tabular}{cc}
\hline Configuration & Number \\
\hline PROFIBUS PA TH400 & 2 \\
PROFINET IO Device & 0 \\
IO controller(S7-300) & 1 \\
Ethernet Switch & 1 \\
Slave IO Data Length & 4 Byte \\
Update Time & $32 \mathrm{~ms} 、 64 \mathrm{~ms}$ \\
\hline
\end{tabular}

Start up. Power up the system, it start up process are shown in Fig.7.
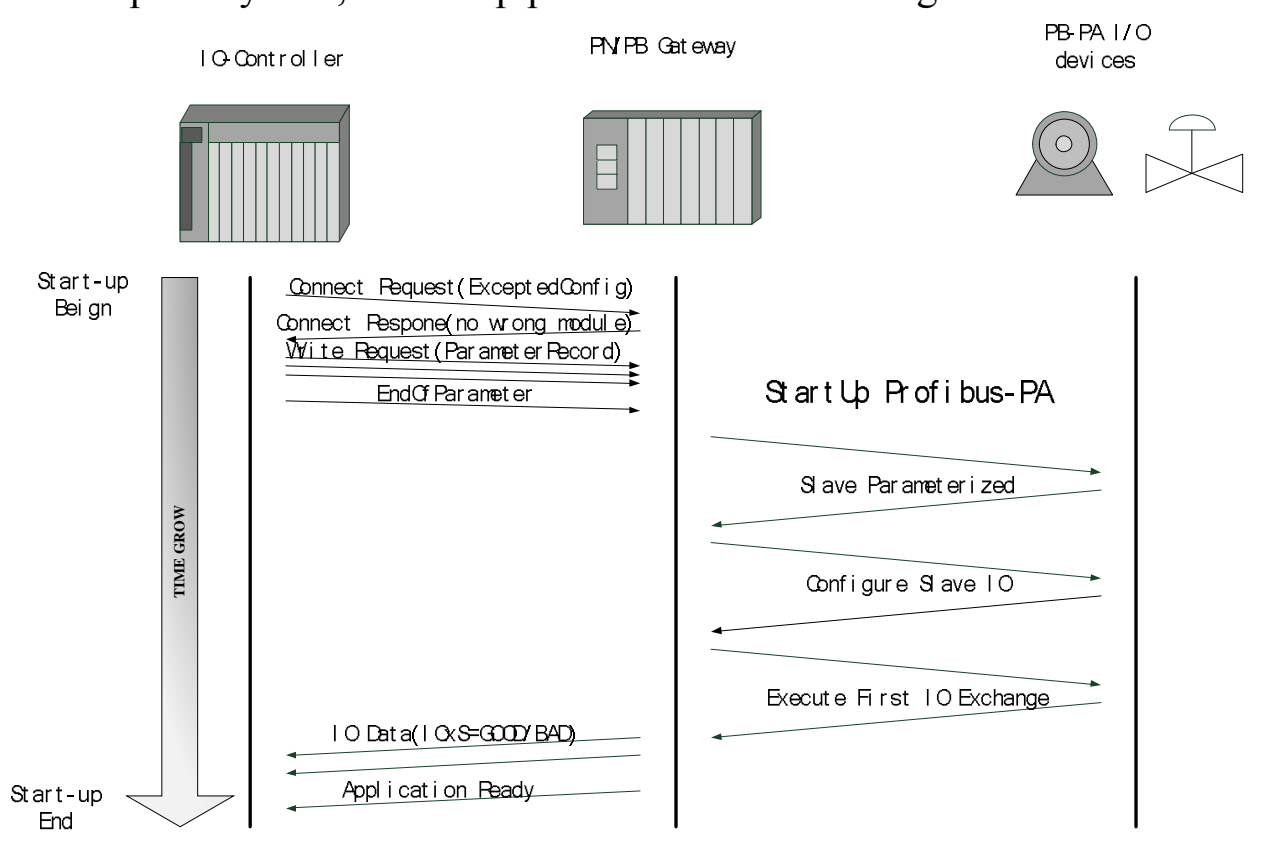

Fig.7 The workflow of system start up

Delay analysis. After system started up we test our gateway's performance for data delay. Due to the different update time of two networks and the cache mode of gateway the delay time may different with output data and input data. The test results are shown in Fig.8. 
Compare input and output delays in the case the gateway's update time is $64 \mathrm{~ms}$, delay time gap between input and output can be found, it should reduce the gap size so that convenient for engineering design. In our more than once test of the gateway update time, the delay gap could be reduce by set the update time value equal to the system response time of PROFIBUS PA system.

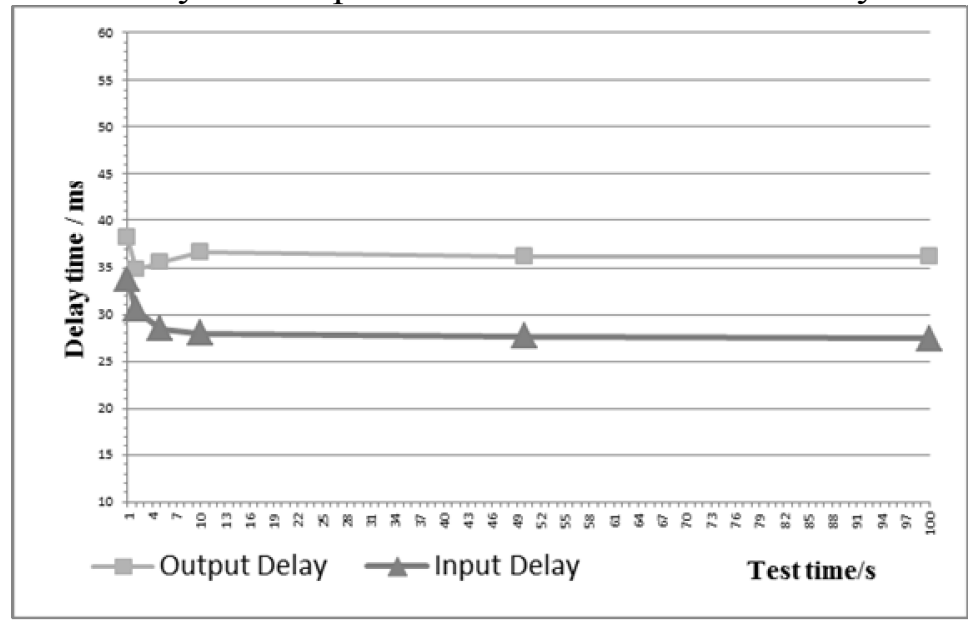

Fig.8 Gateway 64ms update time delay

Considering the process control system application's low real-time requirements, this range of delays are acceptable, gateway performance have satisfied engineering design requirements.

\section{Summary}

Based on the relevant research of PROFIBUS gateway, we design and implement a direct integration system of PROFINET IO and PROFIBUS PA. And we completed functional testing and time delay test, more functionality and performance should be realized in continued study. This design makes the process-oriented industrial fieldbus systems directly integrated into the industrial real-time Ethernet, replacing the traditional couplers connected three layer integrated structure, so that we can take advantage of high speed and openness of industrial Ethernet technology, also make use of the present used system's, simplify network structure of industrial enterprises, accelerate industrial Ethernet technology fully enter into the process industry.

\section{Acknowledgements}

This work was financially supported by the National High Technology Research and Development Program of China (Grant No. 2012AA041701).

\section{References}

[1] Chen Meicheng, Fang Yanjun, Xu Jun. Implementation of fully integrated automation with Profibus[C]. Industrial Electronics Society, 2005. IECON 2005. 31st Annual Conference of IEEE , 2005,11:6-10.

[2] PROFIBUS International. PROFIBUS Specification (Normative Parts of PROFIBUS -FMS, -DP, -PA according to the European Standard)[S]. PROFIBUS International, 1998,3.

[3] Lv Y, Yang ZJ, Duan MQ. A kind of gateway's hardware solution for integrating PROFIBUS PA into PROFINET[C]. 2013 International Conference on Communication Technology, ICCT 2013. Singapore. November 15-16, 2013.

[4] GSD-Spec_2122_V51_Jul08[S]. Karlsruhe Germany:PROFIBUS Nutzerorganisation e.V. Haid-und-Neu-Str. 7 ,July 2008.

[5] SIEMENS.SIMATIC NET GSDML Getting Started Made Easy[M].Germany:Siemens AG, 2009:1-40

[6] Liu Jingyu, Sun Jun, Fang Yanjun. A new approach of integration of PROFIBUS-DP systems into 
industrial Ethernet systems[C]. Control Conference, 2008. CCC 2008. 27th Chinese , 2008,7:550-554. 